OUTP-99??P

\title{
Explicit Calculation Of The Running Coupling BFKL Anomalous Dimension.
}

\author{
Robert S. Thorne \\ Jesus College and Theoretical Physics, \\ University of Oxford, Oxford, Oxon., OX1 3DW, U.K.
}

\begin{abstract}
I calculate the anomalous dimension governing the $Q^{2}$ evolution of the gluon (and structure functions) coming from the running coupling BFKL equation. This may be expressed in an exact analytic form, up to a small ultraviolet renormalon contribution, and hence the corresponding splitting function may be determined precisely. Rather surprisingly it is most efficient to expand the gluon distribution in powers of $\alpha_{s}\left(Q^{2}\right)$ rather than use the traditional expansion where all orders of $\alpha_{s} \ln (1 / x)$ are kept on an equal footing. The anomalous dimension is very different from that obtained from the fixed coupling equation, and leads to a powerlike behaviour for the splitting function as $x \rightarrow 0$ which is far weaker, i.e. $\sim x^{-0.2}$. The NLO corrections to the anomalous dimension are rather small, unlike the fixed coupling case, and a stable perturbative expansion is obtained.
\end{abstract}

December 1999 


\section{Introduction.}

Small $x$ physics has been an active area of theoretical research in the past few years, largely due to the first data for $x<0.005$ being obtained by the HERA experiments [1] [2]. The crux of the debate has been whether the standard DGLAP approach based on renormalization group equations and conventionally ordered simply in powers of $\alpha_{s}\left(Q^{2}\right)$, or the BFKL equation [3], which sums leading logarithms in $(1 / x)$, is most effective, and/or whether the two approaches need to be combined in some way. While the DGLAP approach has been relatively successful, albeit with some significant problems (a valence-like input gluon, undershooting for $x \sim 0.01$ at the highest $Q^{2}$, see [4]), the original BFKL prediction of a behaviour of the form $x^{-\lambda}$ at small $x$, with $\lambda \sim 0.5$, was clearly ruled out. A combination of the two approaches, using the BFKL equation to supplement the Altarelli-Parisi splitting functions with higher terms of the form $\alpha_{s}^{n+1} \ln ^{n}(1 / x)$ had some success (so long as one avoided factorization scheme ambiguities by working in physical quantities) [5], but this was difficult to sustain with the most recent data. Moreover, the subject was thrown into some confusion by the calculation of the NLO correction to the BFKL equation [6][7].

In order to illustrate this I begin with a brief discussion of the LO fixed coupling BFKL equation. Working in moment space, i.e. defining the moment of the structure function by

$$
\mathcal{F}\left(N, Q^{2}\right)=\int_{0}^{1} x^{N-1} F\left(x, Q^{2}\right) d x
$$

and similarly for the parton distributions (scaled by $x$ ), the BFKL equation is

$$
f\left(k^{2}, \bar{\alpha}_{s} / N\right)=f_{I}\left(k^{2}, Q_{0}^{2}\right)+\frac{\bar{\alpha}_{s}}{N} \int_{0}^{\infty} \frac{d q^{2}}{q^{2}} K_{0}\left(q^{2}, k^{2}\right) f\left(q^{2}\right),
$$

where $f\left(k^{2}, \bar{\alpha}_{s} / N\right)$ is the unintegrated gluon four-point function, $f_{I}\left(k^{2}, Q_{0}^{2}\right)$ is the zeroth order input, $\bar{\alpha}_{s}=(3 / \pi) \alpha_{s}$, and the LO kernel is defined by

$$
K_{0}\left(q^{2}, k^{2}\right) f\left(q^{2}\right)=k^{2}\left(\frac{f\left(q^{2}\right)-f\left(k^{2}\right)}{\left|k^{2}-q^{2}\right|}+\frac{f\left(k^{2}\right)}{\left(4 q^{4}+k^{4}\right)^{\frac{1}{2}}}\right) .
$$

It is convenient to define the input by $f_{I}\left(k^{2}, Q_{0}^{2}\right)=\delta\left(k^{2}-Q_{0}^{2}\right)$, where in the case of deep-inelastic scattering, where one end of the gluon ladder is at a hard scale $Q^{2}$, while the other end is formally on-shell, $Q_{0}^{2}$ is just a collinear regularization which we let $\rightarrow 0$ ultimately. The "gluon structure function" is then given by

$$
\mathcal{G}\left(Q^{2}, N\right)=\int_{0}^{Q^{2}} \frac{d k^{2}}{k^{2}} f\left(N, k^{2}, Q_{0}^{2}\right) \times g_{B}\left(N, Q_{0}^{2}\right),
$$

where $g_{B}\left(N, Q_{0}^{2}\right)$ is a bare, nonperturbative gluon density in the proton which implicitly absorbs the collinear divergences in $f\left(k^{2}\right)$. The BFKL equation is most easily solved by taking the Mellin transformation to $\gamma$-space, i.e.

$$
\tilde{f}(\gamma, N)=\int_{0}^{\infty} d k^{2}\left(k^{2}\right)^{-1-\gamma} f\left(k^{2}, N\right)
$$


where it reduces to

$$
\tilde{f}(\gamma, N)=\tilde{f}_{I}\left(\gamma, Q_{0}^{2}\right)+\left(\bar{\alpha}_{s} / N\right) \chi_{0}(\gamma) \tilde{f}(\gamma, N),
$$

where $\tilde{f}\left(\gamma, Q_{0}^{2}\right)=\exp \left(-\gamma \ln \left(Q_{0}^{2}\right)\right)$ and $\chi(\gamma)$ is the characteristic function

$$
\chi_{0}(\gamma)=2 \psi(1)-\psi(\gamma)-\psi(1-\gamma)
$$

A little manipulation leads to the expression

$$
\mathcal{G}\left(Q^{2}, N\right)=\frac{1}{2 \pi i} \int_{\frac{1}{2}-i \infty}^{\frac{1}{2}+i \infty} d \gamma \exp \left(\gamma \ln \left(Q^{2} / Q_{0}^{2}\right)\right) \frac{g_{B}\left(N, Q_{0}^{2}\right)}{\gamma\left(1-\left(\bar{\alpha}_{s} / N\right) \chi_{0}(\gamma)\right)}
$$

This inverse transformation is dominated by the leading pole at $1-\left(\bar{\alpha}_{s} / N\right) \chi_{0}(\gamma)=0$, and the solution is

$$
\mathcal{G}\left(Q^{2}, N\right)=\frac{1}{-\left(\bar{\alpha}_{s} / N\right) \gamma_{0} \chi_{0}^{\prime}\left(\gamma_{0}\right)}\left(\frac{Q^{2}}{Q_{0}^{2}}\right)^{\gamma_{0}} g_{B}\left(N, Q_{0}^{2}\right)
$$

The anomalous dimension $\gamma_{0}\left(\bar{\alpha}_{s} / N\right)$ may be transformed to $x$-space as a power series in $\bar{\alpha}_{s} \ln (1 / x)$, and has a branch point at $N=4 \ln 2 \bar{\alpha}_{s}$ (at which $\gamma \rightarrow \frac{1}{2}$ ) leading to asymptotic small $x$ behaviour for the splitting function

$$
x P^{0}(x) \rightarrow 0.07 \bar{\alpha}_{s} x^{-\lambda} /\left(\bar{\alpha}_{s} \ln (1 / x)\right)^{3 / 2} .
$$

One can, if one ignores the running of the coupling, proceed through exactly the same sort of arguments including the NLO correction to the kernel. In this case the "intercept" for the splitting function is shifted from $\lambda=4 \ln 2 \bar{\alpha}_{s}$ to $\lambda=4 \ln 2 \bar{\alpha}_{s}\left(1-6.5 \bar{\alpha}_{s}\right)$. This is clearly a huge correction, and implies the breakdown of the perturbative expansion for this quantity. Moreover, the power series for the splitting function is dominated by the NLO corrections at all values of $x$ below about $x=0.01$. For example, using the formulae in [6] the first few terms in the power series for $P(x)$ go like

$$
\begin{aligned}
x P(x)= & \bar{\alpha}_{s}+2.4 \bar{\alpha}_{s}^{4} \xi^{3} / 6+2.1 \bar{\alpha}_{s}^{6} \xi^{5} / 120+\cdots \\
& -\bar{\alpha}_{s}\left(0.43 \bar{\alpha}_{s}+1.6 \bar{\alpha}_{s}^{2} \xi+11.7 \bar{\alpha}_{s}^{3} \xi^{2} / 2+13.3 \bar{\alpha}_{s}^{4} \xi^{3} / 6+39.7 \bar{\alpha}_{s}^{5} \xi^{4} / 24+169.4 \bar{\alpha}_{s}^{6} \xi^{5} / 120+\cdots\right),
\end{aligned}
$$

where $\xi=\ln (1 / x)$. Clearly, the size of the coefficients more than compensates for the extra power of $\alpha_{s}$. More careful calculations (discussed a little more below), including the running coupling but using saddle-point approximations imply the same result, but with the coupling being evaluated at $Q^{2}$. This does nothing to aid the convergence, particularly at values of $Q^{2} \sim 1 \mathrm{GeV}^{2}$ where the perturbative analysis of structure function evolution does take place.

Hence, this NLO correction left the whole question of how to address the evolution of structure functions at small $x$ in question, and various alternatives have been proposed to help stabilize the calculation. In this paper I demonstrate that the correct way in which to calculate the $Q^{2}$ evolution at small $x$ is indeed to supplement the conventional AP splitting functions with higher order corrections obtained from the solution to the BFKL equation, but to take the running of the coupling constant fully into account in this equation. The details of this are presented below. 


\section{BFKL Equation for Running Coupling.}

Beyond leading order it is impossible to ignore the running of the coupling, since at NLO ultraviolet regularization is required, resulting in a $\ln \left(k^{2} / \mu_{R}^{2}\right)$ term where $\mu_{R}$ is the renormalization scale. Such a term may be eliminated by using the running coupling constant evaluated at the scale $k^{2}$. Since this is unavoidably forced upon us at NLO, it seems sensible to consider the fixed coupling LO BFKL equation as just a model which would apply in a conformally invariant world, and more realistically to work with the BFKL equation with running coupling [8] [9] from the beginning. Doing this we obtain

$$
f\left(k^{2}, Q_{0}^{2}, \bar{\alpha}_{s}\left(k^{2}\right) / N\right)=f_{I}\left(k^{2}, Q_{0}^{2}\right)+\frac{\bar{\alpha}_{s}\left(k^{2}\right)}{N} \int_{0}^{\infty} \frac{d q^{2}}{q^{2}} K_{0}\left(q^{2}, k^{2}\right) f\left(q^{2}\right),
$$

where

$$
\alpha_{s}=1 /\left(\beta_{0} \ln \left(k^{2} / \Lambda^{2}\right)\right),
$$

$\beta_{0}=\left(11-2 N_{f} / 3\right) /(4 \pi)$, and $N_{f}$ is the number of active flavours. One can solve this equation in the same type of way as for the fixed coupling case, i.e. take the Mellin transformation with respect to $\left(k^{2} / \Lambda^{2}\right)$. It is most convenient first to multiply through by $\ln \left(k^{2} / \Lambda^{2}\right)$, and then obtain

$$
\frac{d \tilde{f}(\gamma, N)}{d \gamma}=\frac{d \tilde{f}_{I}\left(\gamma, Q_{0}^{2}\right)}{d \gamma}-\frac{1}{\bar{\beta}_{0} N} \chi(\gamma) \tilde{f}(\gamma, N),
$$

where $\bar{\beta}_{0}=\left(\pi \beta_{0} / 3\right)$. Hence, the inclusion of the running coupling has completely changed the form of our double Mellin space equation, turning it into a first order differential equation. This has a profound effect on the form of the solutions. The equation may easily be solved giving,

$$
\tilde{f}(\gamma, N)=\exp \left(-X_{0}(\gamma, N) /\left(\bar{\beta}_{0} N\right)\right) \int_{\gamma}^{\infty} \frac{d \tilde{f}_{I}\left(\tilde{\gamma}, N, Q_{0}^{2}\right)}{d \tilde{\gamma}} \exp \left(X_{0}(\tilde{\gamma}) /\left(\bar{\beta}_{0} N\right)\right) d \tilde{\gamma}
$$

where

$$
X_{0}(\gamma)=\int_{\frac{1}{2}}^{\gamma} \chi_{0}(\hat{\gamma}) d \hat{\gamma} \equiv\left(2 \psi(1)\left(\gamma-\frac{1}{2}\right)-\ln \left(\frac{\Gamma(\gamma)}{\Gamma(1-\gamma)}\right)\right)
$$

The leading singularity in the $\gamma$ plane for $\exp \left(-X_{0}(\gamma) /\left(\bar{\beta}_{0} N\right)\right)$, is cancelled by an integral from $0 \rightarrow \gamma$ of the integrand depending on $\tilde{\gamma}$ [11], and so up to $\mathcal{O}\left(\Lambda^{2} / k^{2}\right)$ corrections (2.4) simplifies to

$$
\tilde{f}(\gamma, N)=\exp \left(-X_{0}(\gamma) /\left(\bar{\beta}_{0} N\right)\right) \int_{0}^{\infty} \frac{d \tilde{f}_{I}\left(\tilde{\gamma}, N, Q_{0}^{2}\right)}{d \tilde{\gamma}} \exp \left(X_{0}(\tilde{\gamma}) /\left(\bar{\beta}_{0} N\right)\right) d \tilde{\gamma}
$$

and hence

$$
\begin{aligned}
\mathcal{G}\left(Q^{2}, N\right)= & \frac{1}{2 \pi i} \int_{\frac{1}{2}-i \infty}^{\frac{1}{2}+i \infty} \frac{1}{\gamma} \exp \left(\gamma \ln \left(Q^{2} / \Lambda^{2}\right)-X_{0}(\gamma) /\left(\bar{\beta}_{0} N\right)\right) d \gamma \\
& \times \int_{0}^{\infty} \exp \left(-\tilde{\gamma} \ln \left(Q_{0}^{2} / \Lambda^{2}\right)+X_{0}(\tilde{\gamma}) /\left(\bar{\beta}_{0} N\right)\right) d \tilde{\gamma} g_{B}\left(Q_{0}^{2}, N\right) \\
= & \mathcal{G}_{E}\left(Q^{2}, N\right) \mathcal{G}_{I}\left(Q_{0}^{2}, N\right) g_{B}\left(Q_{0}^{2}, N\right) .
\end{aligned}
$$


The essential expression, $\exp \left(X_{0}(\gamma) /\left(\bar{\beta}_{0} N\right)\right)$, contains singularities at all positive integers, and $\mathcal{G}_{I}\left(Q_{0}^{2}, N\right)$ is not properly defined, since the integrand has singularities lying along the line of integration. These are due to the divergence of the coupling at low $k^{2}$ and can only be removed by some infrared regularization. However, since this factor is independent of $Q^{2}$, it does not contribute at all to the evolution of the structure function, and is irrelevant for the evolution. The function $\mathcal{G}_{E}\left(Q^{2}, N\right)$ is determined by the singularities of $\exp \left(-X_{0}(\gamma) /\left(\bar{\beta}_{0} N\right)\right)$ in the $\gamma$ plane. This leads to a fundamental difference between the cases of the fixed and running couplings. Whereas previously the leading singularity was a pole at $\left(\bar{\alpha}_{s} / N\right) \chi(\gamma)=1$, i.e. at $\gamma \rightarrow \frac{1}{2}$ as $N \rightarrow 4 \ln 2 \bar{\alpha}_{s}$, now the leading singularity is an cut at $\gamma=0$ : there is no powerlike behaviour in $Q^{2}$. Similarly, the branch point in the $N$ plane at $4 \ln 2 \bar{\alpha}_{s}$ has become an essential singularity at $N=0$ : there is no powerlike behaviour in $x$. The introduction of the running of the coupling has changed the character of the solution completely.

Acknowledging that the only real information contained in $\mathcal{G}_{E}\left(N, Q^{2}\right)$ is on the evolution of the structure function, i.e. defining

$$
\frac{d \ln \mathcal{G}\left(N, Q^{2}\right)}{d \ln \left(Q^{2}\right)}=\frac{d \ln \mathcal{G}_{E}\left(N, Q^{2}\right)}{d \ln \left(Q^{2}\right)} \equiv \Gamma\left(N, Q^{2}\right)
$$

$\mathcal{G}_{E}\left(N, Q^{2}\right)$ gives us an entirely perturbative effective anomalous dimension governing the evolution of the gluon structure function. The time-honoured technique for solving for $\mathcal{G}_{E}\left(N, Q^{2}\right)$ is to expand the integrand in (2.7), about the saddlepoint. This results in a contour of integration parallel to the imaginary axis, with real part $\rightarrow \frac{1}{2}$ for the small $x$ solutions. Using this results in an anomalous dimension

$$
\Gamma\left(N, Q^{2}\right)=\gamma_{0}\left(\bar{\alpha}_{s}\left(Q^{2}\right) / N\right)+\sum_{n=1}^{\infty}\left(-\beta_{0} \alpha_{s}\left(Q^{2}\right)\right)^{n} \tilde{\gamma}_{n}\left(\bar{\alpha}_{s}\left(Q^{2}\right) / N\right),
$$

i.e., the effective anomalous dimension is the naive leading order result with coupling at scale $Q^{2}$ plus a series of corrections in increasing powers of $-\beta_{0} \alpha_{s}\left(Q^{2}\right)$. However, each of the $\tilde{\gamma}\left(\bar{\alpha}_{s}\left(Q^{2}\right) / N\right)$ is singular at $N=\lambda\left(Q^{2}\right)$, and the power of the singularity increases with increasing $n$ [12]. Hence, although the series for the resulting splitting function is in the small quantity $\alpha_{s}\left(Q^{2}\right) \beta_{0}$, the accompanying coefficients are progressively more singular as $x \rightarrow 0$. The saddlepoint approximation is therefore not a reliable result as $x \rightarrow 0$ and explicit investigation reveals that it is only really quantitatively useful when $\bar{\alpha}_{s}\left(Q^{2}\right) \ln (1 / x)$ is so small that the effective anomalous dimension is effectively the LO in $\alpha_{s}$ part $x P(x)=\bar{\alpha}_{s}\left(Q^{2}\right)$. Therefore any calculations of the anomalous dimension which rely on an expansion about the saddle-point lead to very inaccurate and misleading results for small $x$.

This instability is not surprising if one examines the integrand along the saddle-point contour of integration, noting that it is very different from the Gaussian form the saddle-point method 
assumes [12], and also if one notes that it is an expansion obtained from approaching $\gamma=\frac{1}{2}$ and in terms of functions of $N$ which are singular at $N=\lambda\left(Q^{2}\right)$, whereas we know that the full solution no longer sees these points as anything special. In fact, the knowledge of the singularity structure of the integrand implies that $\gamma=0$ is a more fruitful point on which to concentrate. Prompted by this we may move the contour of integration to the left and simultaneously use the property that the integrand dies away very quickly at infinity to close the contour so that it simply encloses the real axis for $\gamma<0$. It is then useful to express $\chi_{0}(\gamma)$ in the form

$$
\chi_{0}(\gamma)=1 / \gamma+\sum_{n=1}^{\infty} 2 \zeta(2 n+1) \gamma^{2 n}
$$

which is strictly valid only for $|\gamma|<1$. Doing this we may write

$$
X_{0}(\gamma)=\ln (\gamma)+\gamma_{E}+\sum_{n=1}^{\infty} 2 \frac{\zeta(2 n+1)}{2 n+1} \gamma^{2 n+1}
$$

and the integrand for $\mathcal{G}_{E}\left(N, Q^{2}\right)$ becomes

$$
\gamma^{-1 /\left(\bar{\beta}_{0} N\right)-1} \exp \left(\gamma t-\frac{1}{\left(\bar{\beta}_{0} N\right)}\left(\gamma_{E}+\sum_{n=1}^{\infty} a_{n} \gamma^{2 n+1}\right)\right),
$$

where $t=\ln \left(Q^{2} / \Lambda^{2}\right)$ and $a_{n}=2 \zeta(2 n+1) /(2 n+1)$. The contribution to the integral from $0 \rightarrow-\infty+i \epsilon$ is now the same as that from $-\infty-i \epsilon \rightarrow 0$ up to a phase factor, and we may write

$$
\mathcal{G}_{E}(N, t)=-\sin \left(\frac{\pi}{\left(\bar{\beta}_{0} N\right)}\right) \exp \left(-\frac{\gamma_{E}}{\left(\bar{\beta}_{0} N\right)}\right) \int_{-\infty}^{0} \gamma^{-1 /\left(\bar{\beta}_{0} N\right)-1} \exp \left(\gamma t-\frac{1}{\left(\bar{\beta}_{0} N\right)} \sum_{n=1}^{\infty} a_{n} \gamma^{2 n+1}\right) d \gamma
$$

where the integral has to be understood as an analytic continuation, since there are singularities along the real axis, and strictly speaking the integrand is well defined only for $\gamma>-1$. Since the factor of $\exp (\gamma t)$, is present this latter point leads to an ambiguity of order $\exp (-t)$, i.e. $\mathcal{O}\left(\Lambda^{2} / Q^{2}\right)$ into the value of $\mathcal{G}_{E}(N, t)$.

In order to evaluate the above integral it is convenient to let $y=\gamma t$, resulting in

$$
\begin{aligned}
\mathcal{G}_{E}(N, t)=- & \sin \left(\frac{\pi}{\left(\bar{\beta}_{0} N\right)}\right) \exp \left(-\frac{\gamma_{E}}{\left(\bar{\beta}_{0} N\right)}\right) \\
& t^{1 /\left(\bar{\beta}_{0} N\right)} \int_{-\infty}^{0} y^{-1 /\left(\bar{\beta}_{0} N\right)-1} \exp (y) \exp \left(-\frac{1}{\left(\bar{\beta}_{0} N\right)} \sum_{n=1}^{\infty} a_{n}(y / t)^{2 n+1}\right) d \gamma
\end{aligned}
$$

The latter exponential may be expanded as a power series in $y / t$ and the integral evaluated using the standard result that

$$
(-1)^{n} \Gamma\left(-1 /\left(\bar{\beta}_{0} N\right)+n\right)=\int_{-\infty}^{0} y^{-1 /\left(\bar{\beta}_{0} N\right)-1} \exp (y) y^{n} d \gamma
$$


and hence

$$
\mathcal{G}_{E}(N, t)=-\sin \left(\frac{\pi}{\left(\bar{\beta}_{0} N\right)}\right) \exp \left(-\frac{\gamma_{E}}{\left(\bar{\beta}_{0} N\right)}\right) t^{1 /\left(\bar{\beta}_{0} N\right)} \sum_{n=3}^{\infty} A_{n}\left(1 /\left(\bar{\beta}_{0} N\right)\right) t^{-n}(-1)^{n} \Gamma\left(-1 /\left(\bar{\beta}_{0} N\right)+n\right) .
$$

This result was noted in [13], as was the fact that it may be simplified by using the relationship that as $N \rightarrow 0, \Gamma\left(-1 /\left(\bar{\beta}_{0} N\right)+n\right) \rightarrow \Gamma\left(-1 /\left(\bar{\beta}_{0} N\right)\right)\left(-1 /\left(\bar{\beta}_{0} N\right)\right)^{n}$. However, it is important to notice the more general result that for all $N$

$$
(-1)^{n} \Gamma\left(-1 /\left(\bar{\beta}_{0} N\right)+n\right)=\Gamma\left(-1 /\left(\bar{\beta}_{0} N\right)\right) \Delta_{n}\left(-1 /\left(\bar{\beta}_{0} N\right)\right)
$$

where

$$
\Delta_{n}\left(-1 /\left(\bar{\beta}_{0} N\right)\right)=(-1)^{n} \sum_{m=1}^{n}(-1)^{m} d_{n m}\left(\bar{\beta}_{0} N\right)^{-m},
$$

where $d_{n m}$ are positive coefficients and $d_{n n}=1$. Hence, ignoring the common factor of $-\sin \left(\pi /\left(\bar{\beta}_{0} N\right)\right) \Gamma\left(-1 /\left(\bar{\beta}_{0} N\right)\right) \exp \left(-\gamma_{E} /\left(\bar{\beta}_{0} N\right)\right)$, which has no $t$ dependence, and is irrelevant for the anomalous dimension,

$$
\mathcal{G}_{E}(N, t)=t^{1 /\left(\bar{\beta}_{0} N\right)}\left(1+\sum_{n=3}^{\infty} A_{n}\left(1 /\left(\bar{\beta}_{0} N\right)\right) t^{-n} \Delta_{n}\left(-1 /\left(\bar{\beta}_{0} N\right)\right)\right)
$$

where the $A_{n}$ are simply calculable from the expansion of $\exp \left(-1 /\left(\bar{\beta}_{0} N\right) \sum_{n=1}^{\infty} a_{n}(y / t)^{2 n+1}\right)$. The common factor of $t^{1 /\left(\bar{\beta}_{0} N\right)}$ is the well-known double-leading-log result coming from just the LO $\alpha_{s}\left(Q^{2}\right) / N$ part of the anomalous dimension. Multiplying this we have an expansion as a power series in $1 / t$ or equivalently in $\alpha_{s}\left(Q^{2}\right)$. In fact

$$
t^{-n} \Delta_{n}\left(-1 /\left(\bar{\beta}_{0} N\right)\right)=\left(\bar{\alpha}_{s}\left(Q^{2}\right) / N\right)^{n} \sum_{m=1}^{n} d_{n m}\left(-\beta_{0} \alpha_{s}\left(Q^{2}\right)\right)^{n-m}\left(\bar{\alpha}_{s}\left(Q^{2}\right) / N\right)^{m-n} .
$$

This explicitly demonstrates that we obtain a set of running coupling corrections to a LO result. Substituting this expression for $\mathcal{G}_{E}(N, t)$ in (2.8) one obtains an expression for the anomalous dimension as a power series in $\alpha_{s}\left(Q^{2}\right)$, where at each order we have the leading divergence in $1 / N$ plus a sum of running coupling correction type terms. With a little work one may regain the whole leading $\gamma_{0}\left(\alpha_{s}\left(Q^{2}\right) / N\right)$ (though it is necessary to keep some subleading terms in the $\Delta_{n}$ to do this), along with a tower of terms which are subleading in powers of $\beta_{0} \alpha_{s}\left(Q^{2}\right)$ to this leading anomalous dimension, i.e. one obtains all the corrections to this naive LO anomalous dimension due to the running of the coupling.

The general features of this full, running coupling BFKL anomalous dimension may be appreciated quite easily. The important fact to note is that although the $\left.\Delta_{n}\left(-1 /\left(\bar{\beta}_{0} N\right)\right)\right) \rightarrow\left(1 /\left(\bar{\beta}_{0} N\right)\right)^{n}$ as $N \rightarrow 0$, the function oscillates with $1 /\left(\bar{\beta}_{0} N\right)$, and remains very much smaller in magnitude than 
this asymptotic form until very small $N$, roughly until $1 / N>n$. This coupled with the accompanying factor of $t^{-n}$ means that for reasonable $t$, i.e $t>5\left(Q^{2} \gtrsim 1 \mathrm{GeV}^{2}\right)$, only the first 5 or so terms in (2.19) contribute for $N>0.25$. Hence, to a very good approximation

$$
\mathcal{G}_{E}(N, t)=t^{1 /\left(\bar{\beta}_{0} N\right)}\left(1-\frac{2 \zeta(3)}{3\left(\bar{\beta}_{0} N\right) t^{3}} \Delta_{3}\left(-1 /\left(\bar{\beta}_{0} N\right)\right)-\frac{2 \zeta(5)}{5\left(\bar{\beta}_{0} N\right) t^{5}} \Delta_{5}\left(-1 /\left(\bar{\beta}_{0} N\right)\right)\right)
$$

and in fact the smallness of the coefficient makes even the $t^{-5}$ term almost negligible. $\mathcal{G}_{E}(N, t)$ initially grows as $N$ falls due to the $t^{1 /\left(\bar{\beta}_{0} N\right)}$ term. However, for $N \sim 0.6$ the negative contribution from the $t^{-3}$ term starts to contribute and ultimately drives the gluon structure function to negative values. The result is shown in fig. $1 . d \mathcal{G}_{E}(N, t) / d t$ may simply be evaluated also using (2.21), and shows the same general shape, but does not become negative until a slightly lower value of $N$ as also seen in fig. 1 . Hence the anomalous dimension develops a pole at a finite value of $N$, given quite accurately by

$$
t^{3}=\frac{2 \zeta(3)}{3\left(\bar{\beta}_{0} N\right)}\left(\frac{1}{\left(\bar{\beta}_{0} N\right)^{3}}-\frac{3}{\left(\bar{\beta}_{0} N\right)^{2}}+\frac{2}{\left(\bar{\beta}_{0} N\right)}\right),
$$

where we use the explicit form of $\Delta_{3}\left(-1 /\left(\bar{\beta}_{0} N\right)\right)$. The value of $N$ for this leading pole is shown as a function of $t$ in fig. 2, and for the sort of values relevant at HERA is $\sim 0.25$. Going to $N<0.25$ higher order terms become important, and the positive $1 /\left(\left(\bar{\beta}_{0} N\right)^{2} t^{6}\right) \Delta_{6}\left(-1 /\left(\bar{\beta}_{0} N\right)\right)$ term pulls $\mathcal{G}_{E}(N, t)$ back to positive values, and another pole, with opposite sign residue, appears in $\Gamma(N, t)$. At even lower $N$ the analytic expression eventually breaks down, but numerical results show a series of poles becoming closer together. Nevertheless, the position of the leading pole is essentially determined by the first handful of terms in the power series in $\alpha_{s}\left(Q^{2}\right)$ for $\mathcal{G}_{E}(N, t)$, and hence so is the asymptotic behaviour of the small $x$ splitting function, i.e. $\sim x^{-0.25}$. Hence, the introduction of the running coupling has a dramatic effect on the singularity structure of the LO BFKL anomalous dimension, turning the cut into a series of poles, and changing the position of the rightmost singularity by a factor of $\sim 0.4$. This result of the pole in the anomalous dimension has been already noted in [14] using numerical techniques, and in the context of a resummed NLO anomalous dimension. Here I particularly stress the huge modification of the naive LO BFKL anomalous dimension. This huge change is apparent over a wide range of $N$, and in fig. 3.a I show the anomalous dimension as a function of $N$ for all values right of the leading singularity. As one sees, it is rather closer to the simple $\alpha_{s}\left(Q^{2}\right) / N$ expression than to the naive BFKL result.

Here I should comment on the limit of the analytic expression. As noted, it involves a series expansion not valid over the whole contour of integration. This is reflected in the fact that the overall magnitude of the $\Delta_{n}\left(-1 /\left(\bar{\beta}_{0} N\right)\right)$ increases like $n$ ! in general. This means that the series in (2.21) is actually asymptotic, although it is an oscillating series, so in principle is unambiguously resummable (i.e. the integral in (2.7) does exist). However, the greatest accuracy may be obtained from (2.21) by truncating the series at order $n_{0} \sim t$. In practice I always use $n_{0}=5$. Substituting the truncated expression for $\mathcal{G}_{E}(N, t)$ then results in an infinite series in $\alpha_{s}\left(Q^{2}\right)$ for $\Gamma(N, t)$ which 
is convergent for any given $N$ right of the leading pole. The accuracy of the analytic expression can be found by comparing with results obtained from evaluating (2.7) using numerical integration, and for the gluon structure function for $N$ to the right of the leading pole is found to be rather better than $0.1 \%$ for $t=6$ and falls like $\exp (-t)$.

In order to investigate the quantitative effect of the BFKL anomalous dimension on structure function evolution it is necessary to calculate the BFKL splitting function as a function of $x$. This is where an analytic expression for the anomalous dimension is particularly useful. A series of numerically obtained values of $\Gamma(N, t)$ allows an approximate determination of $P(x, t)$, but it is very difficult to be accurate, especially for the wildly oscillating functions of $1 / N$ which do in fact make up $\mathcal{G}_{E}(N, t)$. However, I now have an explicit series for $\Gamma(N, t)$ in powers of $\alpha_{s}\left(Q^{2}\right)$, obtained from the truncated expression for $\mathcal{G}_{E}(n, t)$. The $N$-dependent functions at each power of $\alpha_{s}\left(Q^{2}\right)$ of course become larger at small $N$ as the series progresses, and to reach small enough $x$ more and more terms are needed. However, at a fixed value of $N$ there is no such growth, and the same is therefore true for fixed $x$. Hence, one only needs to work to a finite order. Limiting oneself to $x>10^{-5}$ and $t>4.5$, the suppression of the $\Delta_{n}\left(-1 /\left(\bar{\beta}_{0} N\right)\right)$ is quite significant and seventh order in $\alpha_{s}\left(Q^{2}\right)$ is easily sufficient. The splitting function for $t=6$ is shown in fig. 4.a. One sees that it is hugely suppressed compared with the naive LO BFKL splitting function, and is even lower than the $\mathcal{O}\left(\alpha_{s}\left(Q^{2}\right)\right)$ contribution for $x$ between about 0.1 and 0.001 . The fact that there is deviation from the standard NLO in $\alpha_{s}\left(Q^{2}\right)$ splitting functions tells us that BFKL influenced structure functions are important. The ultraviolet renormalon contribution is approximated by constructing an $x$-space function which matches the $N$-space results for a variety of $N$. Although this contribution turns out to be a larger fraction of the total than in $N$-space, it still only makes a very small correction to the evolution. This will be discussed more in a subsequent paper [15].

\section{Small $x$ Structure Functions.}

It is important to realize that all of the above results are in a sense ambiguous because they deal with a particular way of defining the gluon parton distribution. It is defined in the natural way for a discussion of BFKL physics, but nonetheless is really an intrinsically factorization-schemedependent quantity. One may define a real structure function by including a hard scattering cross section at the top of the gluon ladder. This modifies (1.4) to

$$
\mathcal{F}_{i}\left(Q^{2}, N\right)=\alpha_{s} \int_{0}^{\infty} \frac{d k^{2}}{k^{2}} \sigma_{i, g}\left(k^{2} / Q^{2}\right) f\left(N, k^{2}, Q_{0}^{2}\right) g_{B}\left(N, Q_{0}^{2}\right) .
$$

Pulling out the overall factor of $\alpha_{s}$ and taking the Mellin transformation of (3.1) with respect to $\left(Q^{2} / \Lambda^{2}\right)$ leads to the simple expression

$$
\tilde{\mathcal{F}}_{i}(\gamma, N)=h_{i, g}(\gamma) \tilde{\mathcal{G}}(\gamma, N)
$$


Thus we may solve for $\mathcal{F}_{i}(N, t)$ in the same way as for $\mathcal{G}(N, t)$ obtaining the same divergent $Q^{2}$ independent part and a $Q^{2}$-dependent part given by solving

$$
\mathcal{F}_{E, i}(N, t)=\frac{1}{2 \pi i} \int_{\frac{1}{2}-i \infty}^{\frac{1}{2}+i \infty} \frac{h_{i, g}(\gamma)}{\gamma} \exp \left(\gamma t-X_{0}(\gamma) /\left(\bar{\beta}_{0} N\right)\right) d \gamma
$$

We may proceed as with the gluon structure function by expanding the $h_{i, g}(\gamma)$ (which were calculated in [16]) as a power series about $\gamma=0$. This results in an expression

$$
\mathcal{F}_{E, i}(N, t)=t^{1 /\left(\bar{\beta}_{0} N\right)}\left(1+\sum_{n=1}^{\infty} B_{i, n}\left(1 /\left(\bar{\beta}_{0} N\right)\right) t^{-n} \Delta_{n}\left(-1 /\left(\bar{\beta}_{0} N\right)\right)\right),
$$

where the $B_{i, n}\left(1 /\left(\bar{\beta}_{0} N\right)\right)$ are now determined not only by the power series in $\gamma$ obtained from the expansion of $X_{0}(\gamma)$, but also from the expansion of $h_{i, g}(\gamma)$. In particular they now contain parts at zeroth order in $1 /\left(\bar{\beta}_{0} N\right)$. In order to draw the most direct analogy to the gluon we define

$$
\Gamma_{L L}(N, t)=\frac{d \ln \left(\mathcal{F}_{L}(N, t)\right)}{d t} .
$$

All the terms in the expression for this physical anomalous dimension, determined from (3.4), are part of the standard LO $\Gamma_{L L}^{0}\left(\alpha_{s}\left(Q^{2}\right) / N\right)$ or are subleading by powers of $\beta_{0} \alpha_{s}\left(Q^{2}\right)$ to this, i.e. again we obtain the naive LO result plus running coupling induced corrections to this, and the "coefficient function" $h_{i, g}(\gamma)$ contributes only to the running coupling corrections.

$\Gamma_{L L}(N, t)$ is shown along with the gluon anomalous dimension in fig. 3.a. Clearly the effect of the additional coefficient function, and hence additional running coupling corrections, is to make $\Gamma_{L L}(N, t)$ dip significantly below the simple $\bar{\alpha}_{s}\left(Q^{2}\right) / N$ and to reduce the value of the intercept compared to the gluon structure function. This is reflected in the effective splitting function $P_{L L}(x, t)$ which is shown in fig. 4.a. This time the dip below the $\mathcal{O}\left(\alpha_{s}\left(Q^{2}\right)\right)$ part is far more pronounced. Also, going to low enough $x$, we see that the splitting function turns over again, showing that the subleading poles in the anomalous dimension may have large residues compared to the leading pole, and the increase in $P_{L L}(x)$ with decreasing $x$ is not monotonic. This illustrates that as far as phenomenology at HERA, or any forseeable collider, is concerned, the value of the intercept for the anomalous dimension is simply not relevant to the evolution of structure functions. I should note that in the case of the physical anomalous dimension and splitting function the power corrections due to the nonconvergence of the series are somewhat larger, about $1 \%$ in $N$-space for $t=6$, and lead to a small, but not insignificant effect on the evolution in $x$-space, and must be accounted for. This will be discussed more in [15].

One can follow exactly the same procedure for the other physical anomalous dimension

$$
\frac{\partial \mathcal{F}_{2}\left(N, Q^{2}\right)}{\partial \ln Q^{2}}=\Gamma_{2 L}\left(Q^{2}, N\right) \mathcal{F}_{L}\left(N, Q^{2}\right),
$$

obtaining qualitatively very similar results. Again the splitting function initially dips as $x$ decreases, but grows again in the same way as $P_{L L}(x, t)$ at lower $x$. This has extremely important implications for the evolution of $F_{2}\left(x, Q^{2}\right)$ in the HERA range, and this will be presented in detail in [15]. 


\section{NLO Corrections.}

So far I have demonstrated that using $\alpha_{s}\left(k^{2}\right)$ in the BFKL equation, as in (2.1), has a profound effect on the form of the solution for the anomalous dimension. However, it is necessary to check that the results presented are not severely modified by the inclusion of the NLO kernel, i.e. the perturbative calculations are stable, and also to justify that the choice of scale in the coupling is correct, or at least leads to accurate results. The NLO kernel was presented in [6] and the way in which to solve at NLO with a running coupling was presented in [17]. Writing the NLO equation as

$$
f\left(k^{2}, Q_{0}^{2}\right)=f_{I}\left(k^{2}, Q_{0}^{2}\right)+\left(\frac{\bar{\alpha}_{s}\left(k^{2}\right)}{N}\right) \int_{0}^{\infty} \frac{d q^{2}}{q^{2}}\left(K_{0}\left(q^{2}, k^{2}\right)-\alpha_{s}\left(k^{2}\right) K_{1}\left(q^{2}, k^{2}\right)\right) f\left(q^{2}\right),
$$

using just the one-loop coupling leads to a 2 nd order differential equation in $\gamma$-space

$$
\frac{d^{2} \tilde{f}(\gamma, N)}{d \gamma^{2}}=\frac{d^{2} \tilde{f}_{I}\left(\gamma, Q_{0}^{2}\right)}{d \gamma^{2}}-\frac{1}{\bar{\beta}_{0} N} \frac{d\left(\chi_{0}(\gamma) \tilde{f}(\gamma, N)\right)}{d \gamma}-\frac{\pi}{3 \bar{\beta}_{0}^{2} N} \chi_{1}(\gamma) \tilde{f}(\gamma, N)
$$

This can be solved in a very similar way to LO, i.e. it factorizes into the same form as (2.7) with $Q^{2}$-dependent part given by

$$
\mathcal{G}_{E, N L O}(N, t)=\frac{1}{2 \pi i} \int_{\frac{1}{2}-i \infty}^{\frac{1}{2}+i \infty} \frac{1}{\gamma} \exp \left(\gamma t-X_{N L O}(\gamma, N) /\left(\bar{\beta}_{0} N\right)\right) d \gamma
$$

However, $X_{N L O}(\gamma, N)$ is rather more complicated than the previous $X_{0}(\gamma)$. It can be expressed in the form

$$
X_{N L O}(\gamma, N)=\int_{\frac{1}{2}}^{\gamma} \chi_{N L O}(\hat{\gamma}, N) d \hat{\gamma}
$$

where $\chi_{N L O}(\gamma, N)$ can be written as a power series in $N$ beginning at zeroth order with $\chi_{0}(\gamma)$. As seen in [17], though here ignoring any resummations in $N$, the explicit form is

$$
\chi_{N L O}(\gamma, N)=\chi_{0}(\gamma)-N \frac{\chi_{1}(\gamma)}{\chi_{0}(\gamma)}+\frac{N^{2}}{\chi_{0}}\left(-\left(\frac{\chi_{1}(\gamma)}{\chi_{0}(\gamma)}\right)^{2}-\beta_{0}\left(\frac{\chi_{1}(\gamma)}{\chi_{0}(\gamma)}\right)^{\prime}\right)+\cdots
$$

where $\chi_{2}(\gamma)$ would also appear at order $N^{2}$, had I included it. I shall generally ignore all but the first two terms.

Firstly, I shall address the choice of scale. It was known in [18] that the correct scale was really $(k-q)^{2}$, but that $k^{2}$ could be used, leading to a part of the NLO kernel which is proportional to $\beta_{0}$, i.e. there is a contribution to $\chi_{1}(\gamma)$ of the form $\frac{1}{2} \bar{\beta}_{0}\left(\chi_{0}^{2}(\gamma)+\chi_{0}^{\prime}(\gamma)\right)$. Substituting this into (4.3) leads to a contribution in the integrand of the form $\exp \left(\frac{1}{2}\left(\ln \left(\chi_{0}(\gamma)\right)+X_{0}(\gamma)\right)\right)$. This can be expressed as a power series which at low orders is $1+1.6 \gamma^{3}+1.24 \gamma^{5}$. Hence, this scale-induced factor has the same form as the $h_{i, g}(\gamma)$, and not surprisingly results in additional running coupling corrections to the anomalous dimensions. However, the terms in the series do not start until third 
order, have small coefficients, and have an effect much smaller than $h_{i, L(2)}(\gamma)$. Hence, the correction for this "incorrect" choice of scale is very small, though in principle it seems as though the factor just considered should really be taken as part of the LO result, since it just gives running coupling corrections only. ${ }^{1}$

So the choice of $\alpha_{s}\left(k^{2}\right)$ is in practice very reliable, and may easily be corrected for. We must now consider the rest of the NLO correction to the kernel, which is much larger. Here we have an ambiguity in precisely what the NLO calculation means. Do we simply solve (4.1), producing the infinite series in (4.5)? Do we truncate $\chi_{N L O}(\gamma, N)$ after the second term, and if so do we use the whole of $\exp \left(1 / \bar{\beta}_{0} \int_{\frac{1}{2}}^{\gamma}\left(\chi_{1}(\hat{\gamma}) / \chi_{0}(\hat{\gamma})\right) d \hat{\gamma}\right)$ or just expand it out to first order? There are particular problems associated with all choices. I choose the NLO definition such that the anomalous dimension receives only corrections which are one power of $\alpha_{s}\left(Q^{2}\right)$ down on the leading order one, i.e. the LO anomalous dimension is of the form $\Gamma^{0}\left(\bar{\alpha}_{s}\left(Q^{2}\right) / N, \beta_{0} \alpha_{s}\left(Q^{2}\right)\right)$ and the NLO corrected one is of the form $\Gamma^{0}\left(\bar{\alpha}_{s}\left(Q^{2}\right) / N, \beta_{0} \alpha_{s}\left(Q^{2}\right)\right)+\alpha_{s}\left(Q^{2}\right) \Gamma^{1}\left(\bar{\alpha}_{s}\left(Q^{2}\right) / N, \beta_{0} \alpha_{s}\left(Q^{2}\right)\right)$. Roughly speaking, this involves keeping only the first two terms in (4.5), and expanding $\exp \left(1 / \bar{\beta}_{0} \int_{\frac{1}{2}}^{\gamma}\left(\chi_{1}(\hat{\gamma}) / \chi_{0}(\hat{\gamma})\right) d \hat{\gamma}\right)$, out to just first order in $1 / \bar{\beta}_{0}$. However, the part of $\left(\chi_{1}(\hat{\gamma}) / \chi_{0}(\hat{\gamma})\right)$ behaving like $1 / \gamma$ must be treated very carefully. Details will be presented in [15].

Solving for the NLO anomalous dimension using the same techniques as at LO results in the NLO correction. Unlike the case of fixed coupling, or the naive results of the saddle-point evaluation, these corrections are rather small. The positions of the leading poles in the anomalous dimensions are shown in fig. 2 , and one can see that they change from about 0.21 for $\Gamma_{L L}$ at LO to 0.16 at NLO, and that the $Q^{2}$-dependence reduces a little. Similarly the anomalous dimension $\Gamma_{L L}(N, t)$ over a wide range of $N$ shows only a very small change going from LO to NLO. (I use the physical anomalous dimensions to avoid any ambiguity. The results are very similar for the gluon distribution.) This is shown in fig. 3.b where the part of the NLO anomalous dimension at first order in $\alpha_{s}\left(Q^{2}\right)$, i.e $-0.935 \alpha_{s}\left(Q^{2}\right)$, is not included, since this should properly be included at $\mathrm{LO}$ in a combined leading order in $\alpha_{s}\left(Q^{2}\right)$ and $\alpha_{s}\left(Q^{2}\right) \ln (1 / x)$ expansion scheme. Alternative definitions of NLO lead to very similar results except at very high values of $N$. Note that the NLO correction is actually positive for $N \sim 0.9$ - very different from the case where running coupling corrections are not included and all NLO corrections are negative. I should also note that the ultraviolet renormalon contribution is a far larger proportion of the NLO correction than it is of the LO contribution, being typically $10 \%$ for $t=6$, and needs to be accounted for [15].

One can also make the transformation to $x$-space and calculate the NLO corrected splitting function. This is shown for $t=6$ in fig. 4.b, where the contributions $\propto \delta(1-x)$ both from the $\mathcal{O}\left(\alpha_{s}\left(Q^{2}\right)\right)$ part and the running coupling corrections to this are absent. The latter of these is a

1 In fact, the anomalous dimensions and splitting functions already presented in this paper contain corrections from this factor, though this makes only a very small difference. 
very small contribution. The NLO corrected splitting function is not too different from that at LO, as one can see. However, the real import of the NLO corrections as far as physics is concerned is the effect it has on the evolution of the structure function. This is demonstrated in fig. 5 where the evolution of a suitable model for the structure function $F_{L}\left(x, Q^{2}\right)$, i.e. $(1-x)^{6} x^{-0.2}$, is shown both for the LO running coupling splitting function, and for the NLO corrected one (all $\delta(1-x)$ contributions other that at first order in $\alpha_{s}\left(Q^{2}\right)$ one are included). As one sees, at this (rather low) value of $t$, i.e. $Q^{2} \sim 8 \mathrm{GeV}^{2}$, the effect of the $\mathrm{NLO}$ corrections is only of order $10 \%$, and is positive for $x \sim 0.01$. Also shown for comparison is the contribution from the $\bar{\alpha}_{s}\left(Q^{2}\right) / x$ term alone. The running coupling BFKL splitting function leads to slightly quicker evolution than this latter contribution for $x \gtrsim 0.01$, but for $x \sim 0.0001$ the evolution is significantly suppressed.

Hence, the NLO corrections to the running coupling BFKL derived splitting function are well under control, both in terms of the asymptotic powerlike behaviour of the splitting functions and in terms of the evolution in the range currently accessible to experiments. Beyond the running coupling corrections no further resummations are necessary, or even useful. This is very much in contrast to the case where both ends of the gluon ladder are associated with a hard scale, the so-called "single scale" processes. In this case, as shown in [19] and developed in [17] and [14], the conventional BFKL expansion is fundamentally flawed due to high order poles at $\gamma=0$ and 1 , which need to be resummed. Without resummation, all calculations are badly behaved over the full range of $N$, not just at low $N$. In the case of deep inelastic scattering the collinear factorization procedure automatically orders the poles at $\gamma=0$ correctly, and the above problem shows up in high order poles at $\gamma=1$ only. The anomalous dimension is totally dominated by the region very close to $\gamma=0$, as this paper shows, and is very insensitive to effects at $\gamma=1$. Including the type of resummation in [19][17] alters results from the NLO corrected case by a very small amount, and is possibly no more influential than the remaining NNLO effects for which it does not account. The corrected treatment at $\gamma=1$ is essential if one is attempting to obtain information about the input form of the gluon, i.e. $\mathcal{G}_{I}\left(Q_{0}^{2}, N\right)$, but this, along with the whole subject of single-scale processes, is also plagued by the infrared divergence problem. A discussion of such issues can be found in $[14][20]$.

\section{Conclusions.}

I have shown that an analytic expression for the anomalous dimensions and splitting functions obtained from the running coupling BFKL equation may be obtained by a power series solution for the $Q^{2}$-dependent part of the gluon structure function in terms of $\alpha_{s}\left(Q^{2}\right)$. This has extremely good accuracy, with only very small errors, which may be interpreted as ultraviolet renormalon contributions, and may be calculated numerically. Moreover, I find the remarkable result that the form of this anomalous dimension and splitting function is almost completely determined by only 
the first handful $(\sim 5)$ of terms in the expansion for the gluon. This is in complete contrast with the case of fixed coupling, where an all orders summation is needed, and it would be interesting to understand the origin of this phenomena.

My results also prove that the effect of the running of the coupling is to weaken the asymptotic powerlike growth of the splitting functions severely compared to the naive results, and even to lower the splitting function below the $\alpha_{s}\left(Q^{2}\right) / x$ contribution for $0.001 \gtrsim x \gtrsim 0.2$. It also makes the NLO correction to the splitting functions relatively small, both for the value of the intercept and for the evolution of structure functions for $x>10^{-5}$, and therefore stabilizes the perturbative series. I also note that a previous conjecture that the effect of the running coupling in the BFKL equation could be accounted for using an $x$-dependent scale for the coupling [12], resulting in falling coupling for decreasing $x$, turns out to be largely correct so long as the change in the scale of the coupling is moderate compared to the scale itself, though it fails when this criterion is not satisfied. In practice this condition is identical to that specifying that diffusion in the fixed coupling BFKL equation is not too large and therefore that the virtualities sampled in the running coupling equation are not too far away from $Q^{2}$. This results in the requirement that $t^{3} \gtrsim 20 \ln (1 / x)$ [21]. This covers most of the HERA range for $Q^{2}>1 \mathrm{GeV}^{2}$, and means that a phenomenological analysis using the explicit resummation in this paper leads to very similar results to the approach in [12]. In particular, the input $F_{L}\left(x, Q_{0}^{2}\right)$ is of the same shape as $F_{2}\left(x, Q_{0}^{2}\right)$ for $Q_{0}^{2}$ as low as $1 \mathrm{GeV}^{2}$, in contrast to the standard NLO-in- $\alpha_{s}\left(Q^{2}\right)$ approach, and the quality of the fit, particularly for small $x$ data, is far better than using the conventional approach. A more detailed presentation of such

a phenomenological study will appear. However, I can say that the inclusion of corrections to the fixed order in $\alpha_{s}\left(Q^{2}\right)$ splitting functions from the running coupling BFKL equation not only leads to a stable perturbative expansion but also to a clear improvement in the comparison to data.

\section{Acknowledgements.}

I would like to thank A.D. Martin, A.H. Mueller, R.G. Roberts, D.A. Ross and W.J. Stirling for useful discussions. 


\section{Figure Captions}

Fig. 1. The $Q^{2}$-dependent part of the gluon structure function, $\mathcal{G}_{E}(N, t)$, and $d \mathcal{G}_{E}(N, t) / d t$ as a function of $N$ for $t=6\left(Q^{2} \sim 8 \mathrm{GeV}^{2}\right)$. The $Q^{2}$-independent factor of $-\sin \left(\pi /\left(\bar{\beta}_{0} N\right)\right) \Gamma\left(-1 /\left(\bar{\beta}_{0} N\right)\right) \exp \left(-\gamma_{E} /\left(\bar{\beta}_{0} N\right)\right)$ is included in both in order to produce a more smoother $N$-dependent normalization of the functions.

Fig. 2. The positions of the leading poles in the anomalous dimensions for the gluon structure function at LO, for $F_{L}$ at $\mathrm{LO}$ and for $F_{L}$ at NLO.

Fig. 3. a. The anomalous dimensions for the gluon structure function at LO and for $F_{L}$ at LO plotted as functions of $N$ for $t=6\left(Q^{2} \sim 8 \mathrm{GeV}^{2}\right)$. Also shown is the $\mathcal{O}\left(\alpha_{s}\left(Q^{2}\right)\right)$ contribution $\bar{\alpha}_{s}\left(Q^{2}\right) / N$. b. The anomalous dimensions for for $F_{L}$ at $\mathrm{LO}$ and at NLO plotted as functions of $N$ for $t=6$.

Fig. 4. a. The splitting functions $x P_{g g}(x)$ and $x P_{L L}(x)$ plotted as a function of $x$ for $t=6$ $\left(Q^{2} \sim 8 \mathrm{GeV}^{2}\right)$. Also shown is the $\mathcal{O}\left(\alpha_{s}\left(Q^{2}\right)\right)$ contribution $\bar{\alpha}_{s}\left(Q^{2}\right)$, and the naive LO BFKL splitting function with coupling $\alpha_{s}\left(Q^{2}\right)$. b. The splitting functions $x P_{L L}^{L O}(x)$ and $x P_{L L}^{N L O}(x)$ plotted as a function of $x$ for $t=6$.

Fig. 5. The values of $d F_{L}\left(x, Q^{2}\right) / d \ln Q^{2}$, for $F_{L}\left(x, Q^{2}\right)=x^{-0.2}(1-x)^{6}$, due to the LO splitting functions $P_{L L}^{L O}(x)$ and the NLO splitting function $P_{L L}^{N L O}(x)$, plotted as a function of $x$ for $t=6\left(Q^{2} \sim 8 \mathrm{GeV}^{2}\right)$. Also shown is the evolution due to the $\mathcal{O}\left(\alpha_{s}\left(Q^{2}\right)\right)$ contribution $P(x)=\bar{\alpha}_{s}\left(Q^{2}\right) / x$. 


\section{References}

[1] H1 collaboration: S. Aid et al.,Nucl. Phys. B470 (1996) 3;

H1 collaboration: C. Adloff et al., Nucl. Phys. B497 (1997) 3;

H1 collaboration: C. Adloff et al., hep-ex/9908059.

[2] ZEUS collaboration: M. Derrick et al, Zeit. Phys. C69 (1996) 607;

ZEUS collaboration: M. Derrick et al, Zeit. Phys. C72 (1996) 399.

[3] L.N. Lipatov, Sov. Jour. Nucl. Phys. 23 (1976) 338;

E.A. Kuraev, L.N. Lipatov and V.S. Fadin, Sov. Jour. JETP 45 (1977) 199;

Ya. Balitskii and L.N. Lipatov, Sov. Jour. Nucl. Phys. 28 (1978) 6.

[4] A.D. Martin et al, Eur. Jour. Phys., C4 (1998) 463.

[5] R.S. Thorne, Phys. Lett. B392 (1997) 463; Nucl. Phys. B512 (1998) 323.

[6] V.S. Fadin and L.N. Lipatov, Phys. Lett. B429 (1998) 127, and references therein.

[7] G. Camici and M. Ciafaloni, Phys. Lett. B430 (1998) 349, and references therein.

[8] L.V. Gribov, E.M. Levin and M.G. Ryskin, Phys. Rep. 100 (1983) 1.

[9] L.N. Lipatov, Sov. Phys. JETP 63 (1986) 904.

[10] J. Kwiecinski, Zeit. Phys. C29 (1985) 561.

[11] J.C. Collins and J. Kwiecinski, Nucl. Phys. B316 (1989) 307.

[12] R.S. Thorne, Phys. Rev. D60 (1999) 054031.

[13] A.D. Martin and J. Kwiecinski, Phys. Lett. B353 (1995) 123.

[14] M. Ciafaloni, D. Colferai and G.P. Salam, Phys. Rev. D60 (1999) 114036.

[15] R. S. Thorne, in preparation.

[16] S. Catani and F. Hautmann, Phys. Lett. B315 (1993) 157; Nucl. Phys. B427 (1994) 475.

[17] M. Ciafaloni and D. Colferai, Phys. Lett. 452 (1999) 372.

[18] G. Camici and M. Ciafaloni, Phys. Lett. B386 (1996) 341.

[19] G.P. Salam, JHEP 9807 (1998) 19.

[20] M.Ciafaloni, D. Colferai and G.P. Salam, JHEP 9910 (1999) 017.

[21] A.H. Mueller, Phys. Lett. B396 (1997) 251. 
$\mathrm{Q}^{2}$-dependent gluon and its $\ln \mathrm{Q}^{2}$-derivative

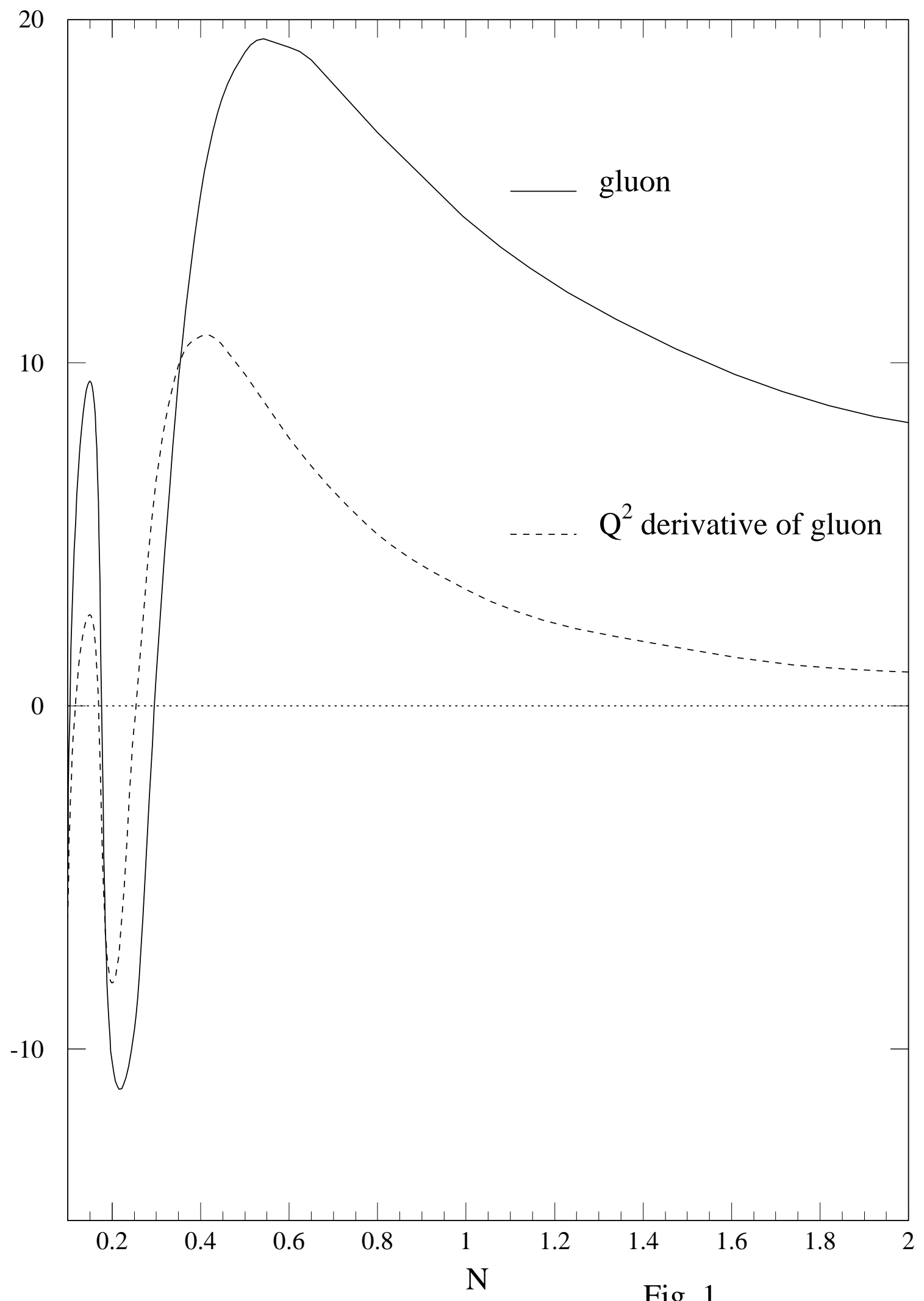

Fig. 1 
Comparison of Intercepts

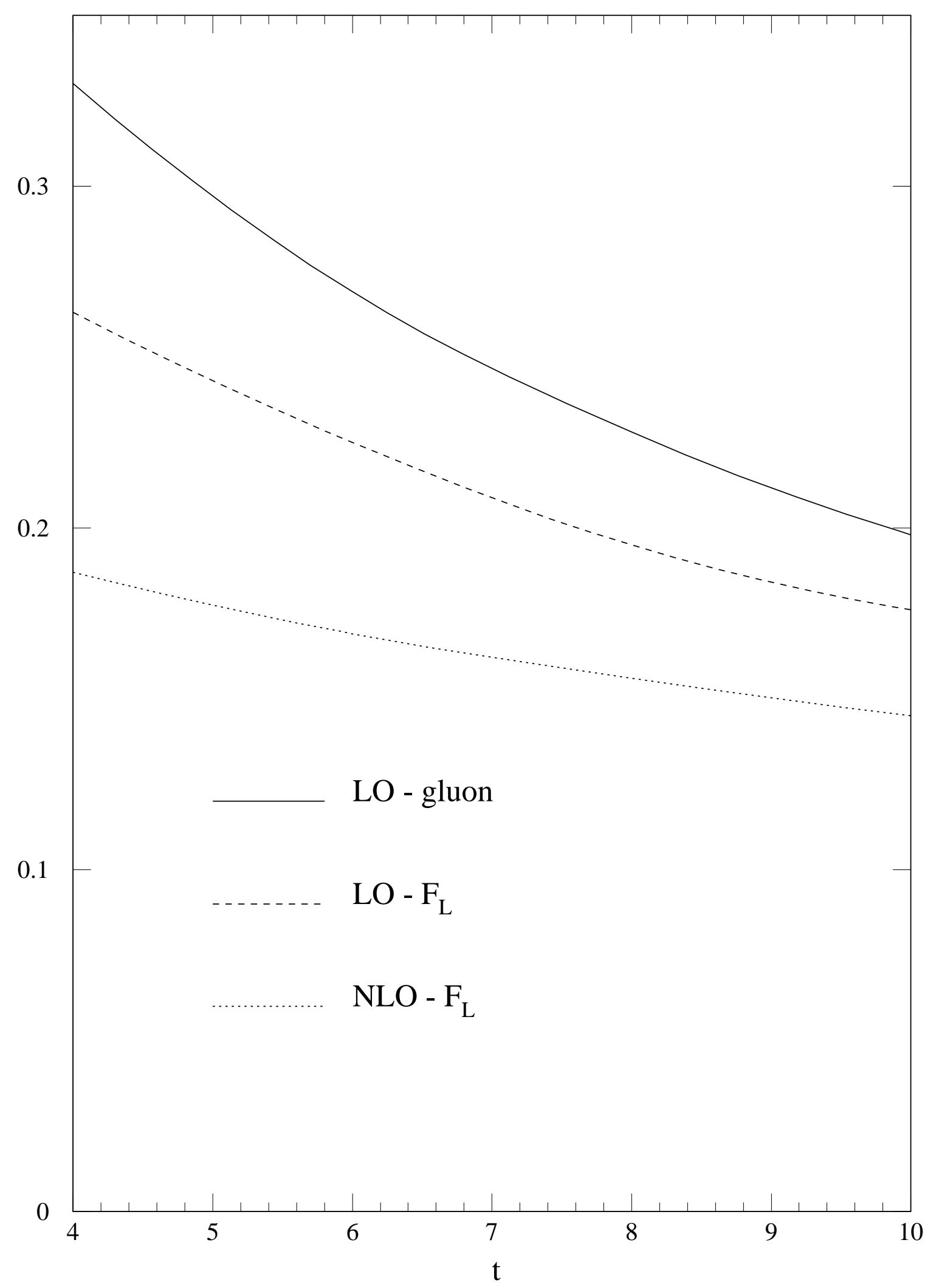

Fig. 2 


\section{Comparison of Anomalous Dimensions}

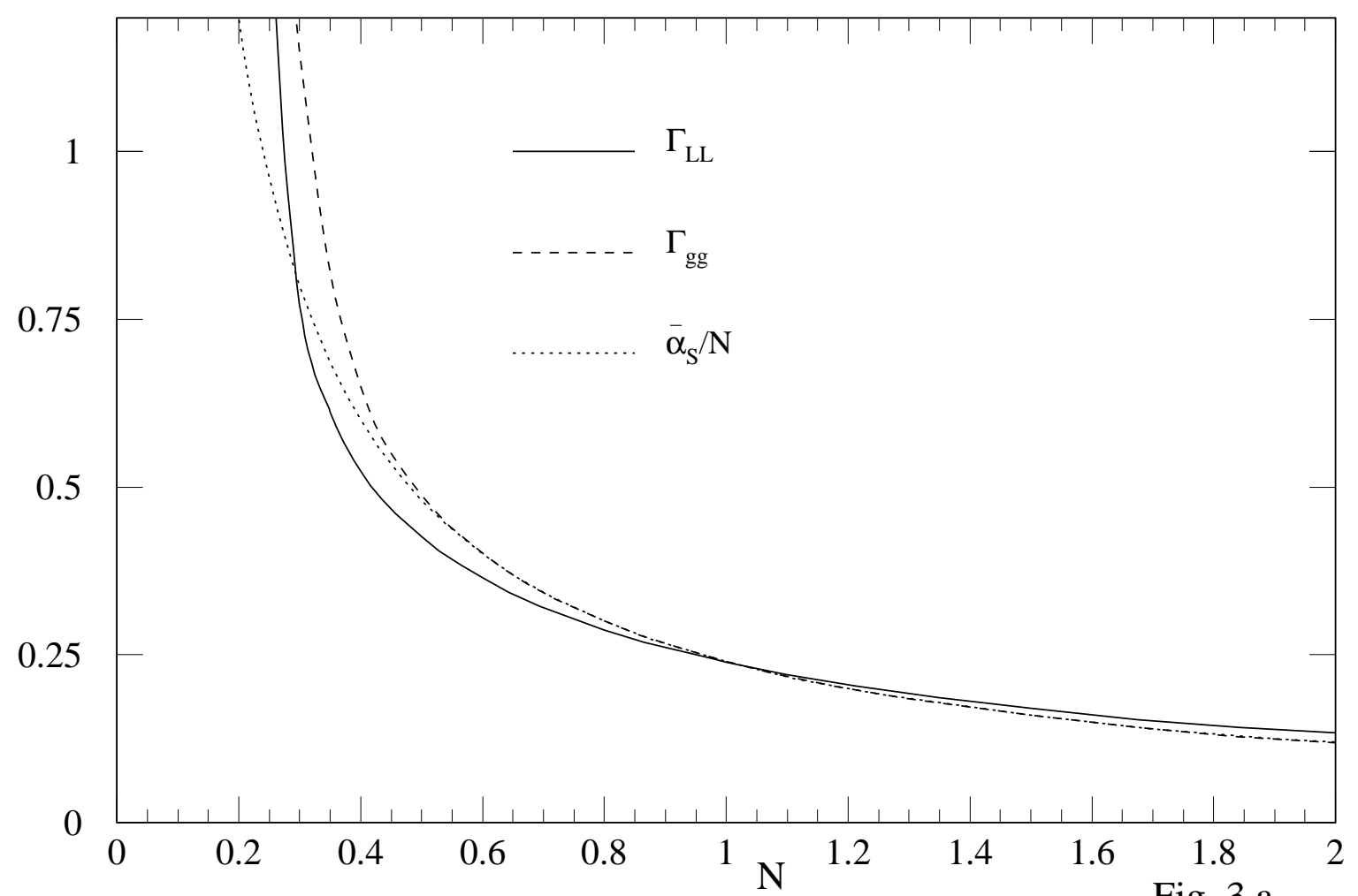

Fig. 3.a

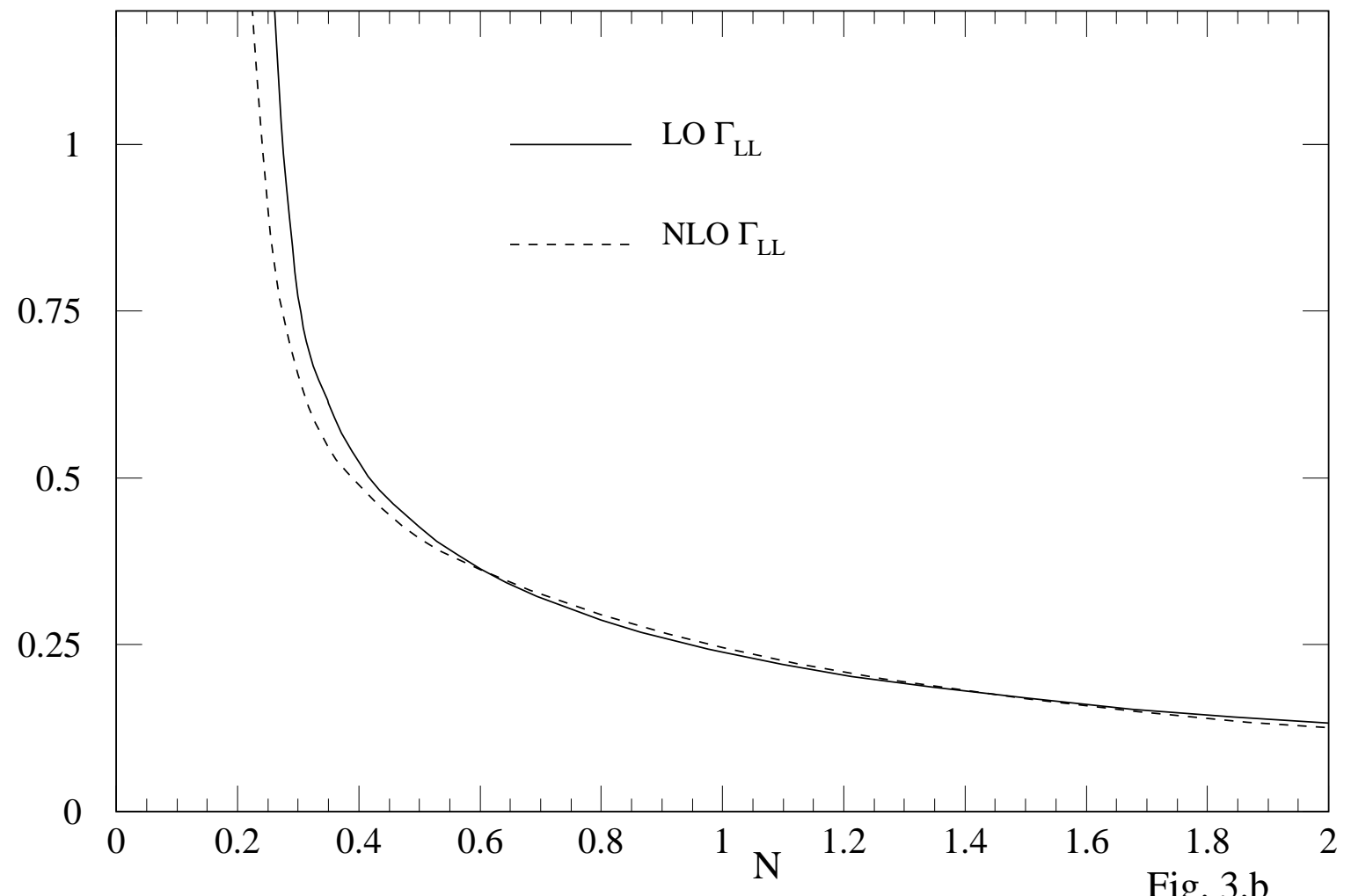

Fig. 3.b 
Comparison of Splitting Functions

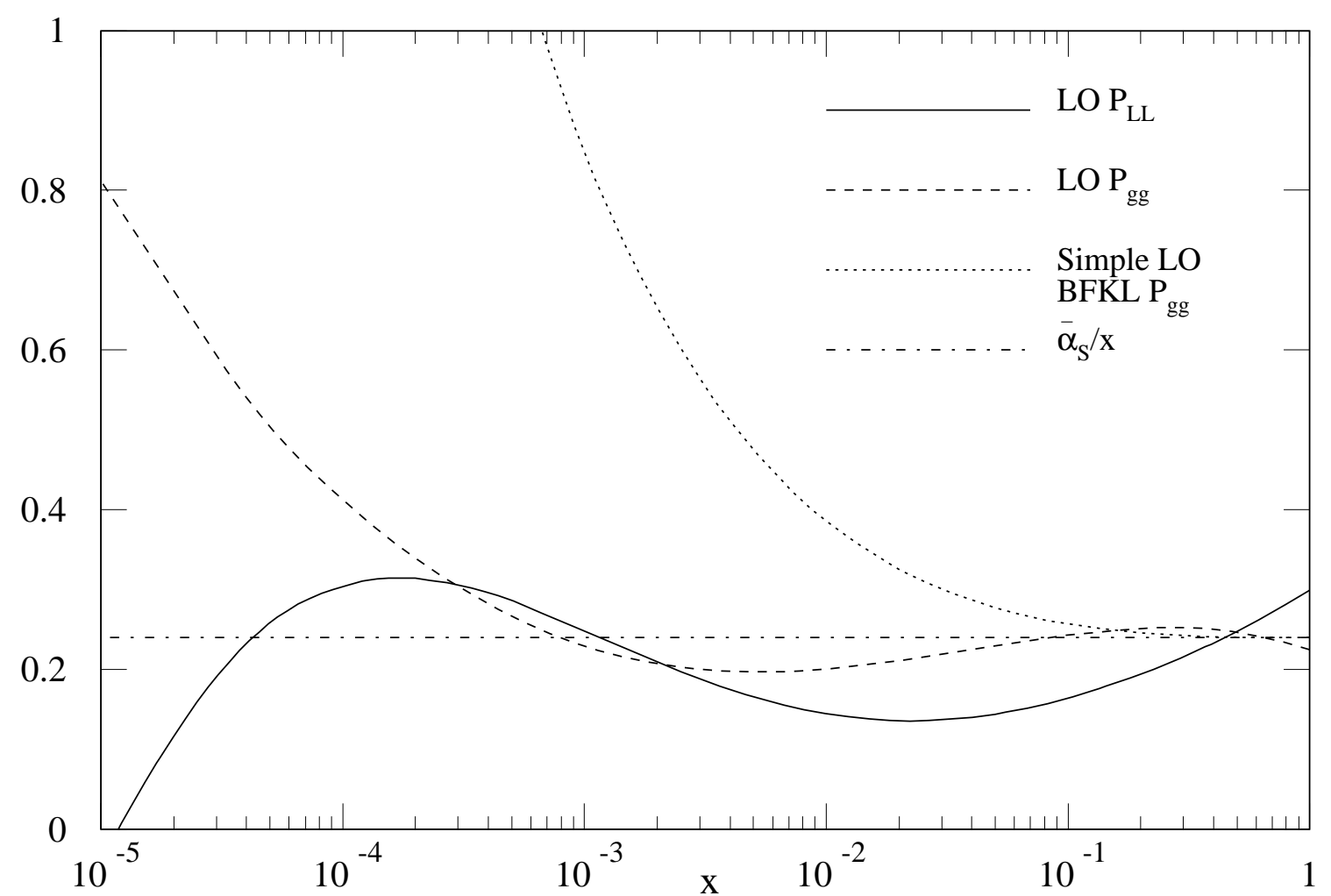

Fig. 4.a

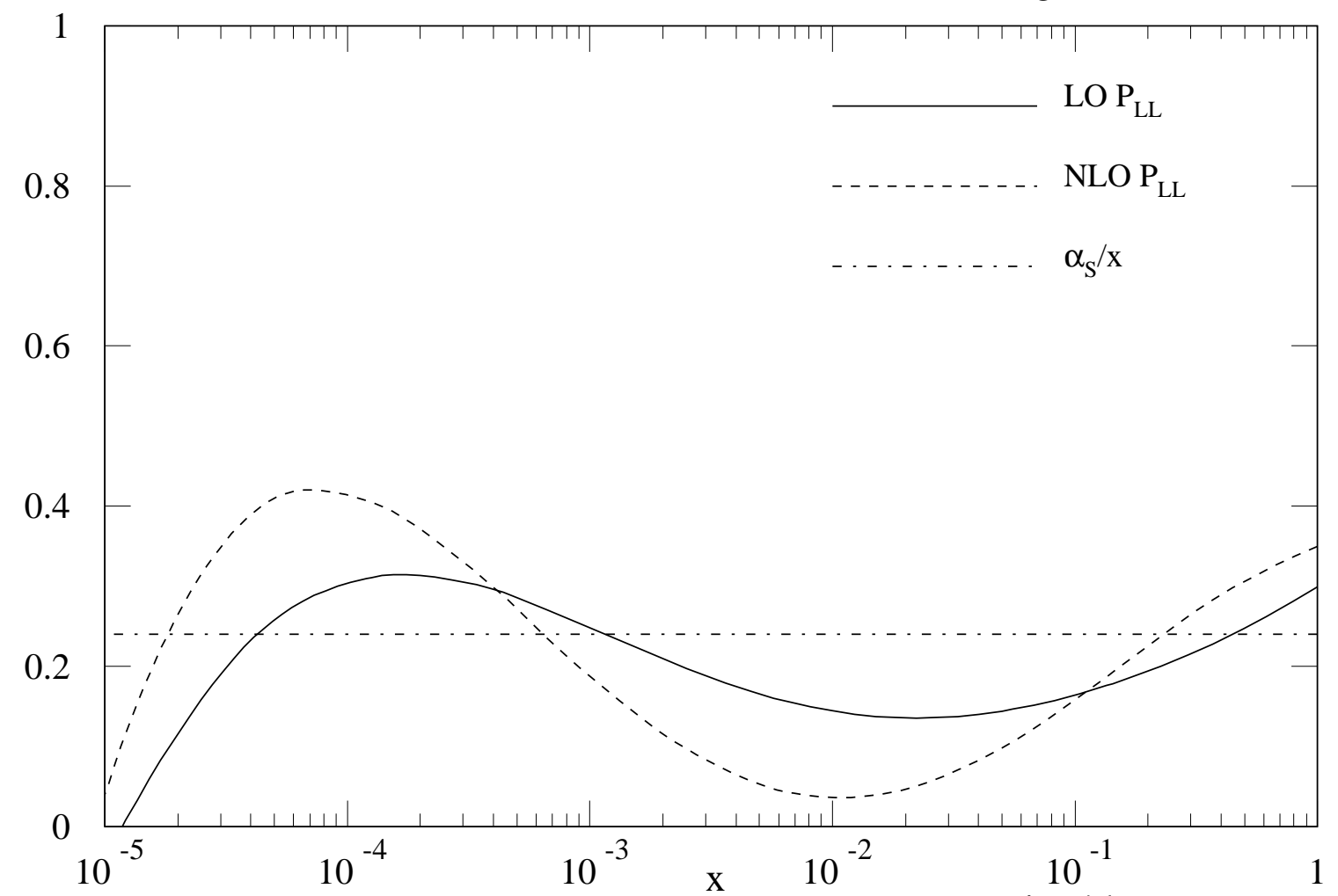

Fig. 4.b 
Evolution of $F_{L}, \ln \left(Q^{2} / \Lambda^{2}\right)=6, F_{L}=(1-x)^{6} x^{-0.2}$

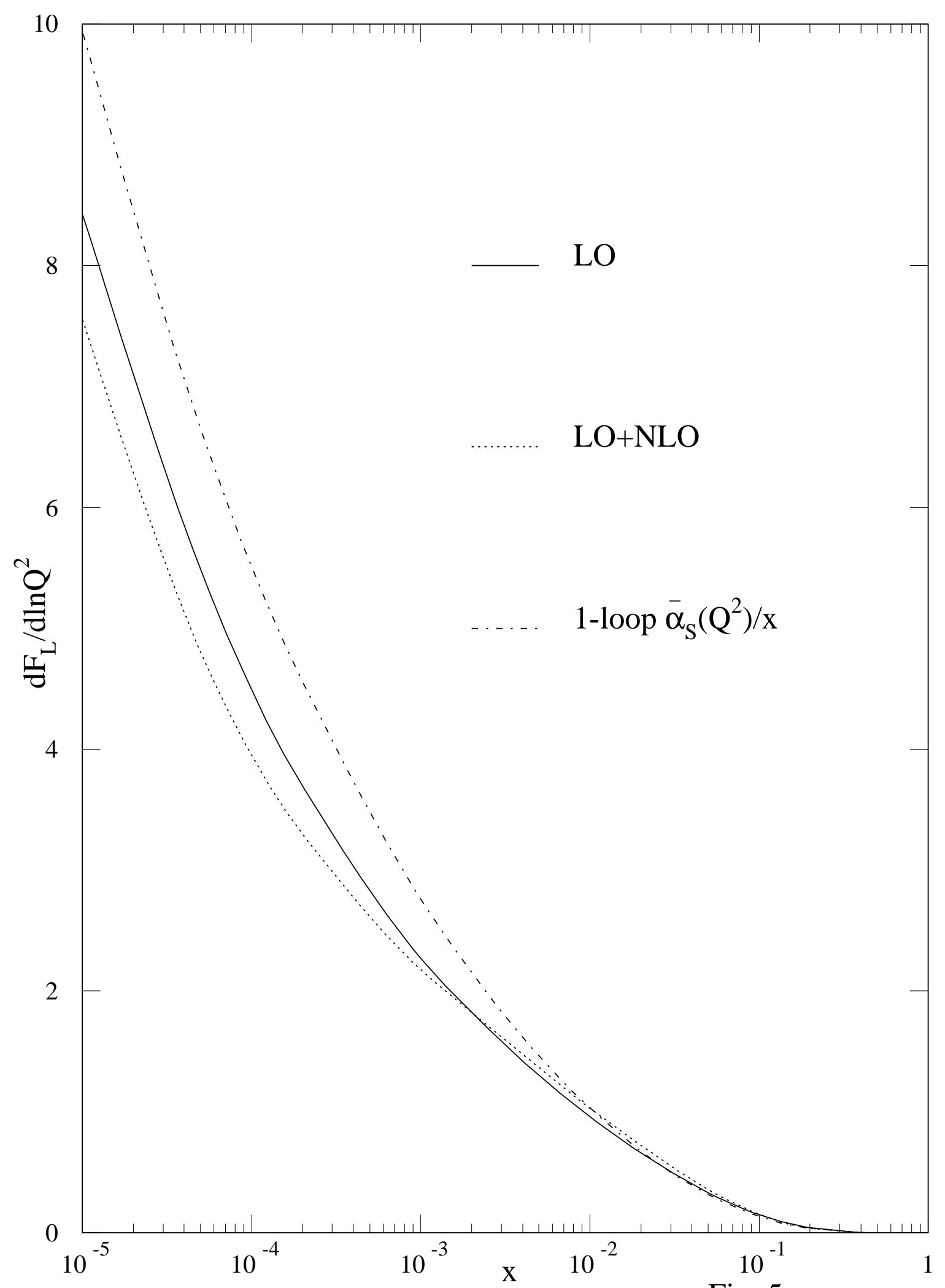

Fig. 5 